\title{
Patterning Electro-osmotic Flow with Patterned Surface Charge
}

\author{
Abraham D. Stroock, Marcus Weck, Daniel T. Chiu, Wilhelm T. S. Huck, Paul J. A. Kenis, \\ Rustem F. Ismagilov, and George M. Whitesides* \\ Department of Chemistry and Chemical Biology, Harvard University, Cambridge, Massachusetts 02138
}

(Received 2 November 1999)

This Letter reports the measurement of electro-osmotic flows (EOF) in microchannels with surface charge patterned on the $200 \mu \mathrm{m}$ scale. We have investigated two classes of patterns: (1) Those in which the surface charge varies along a direction perpendicular to the electric field used to drive the EOF; this type of pattern generates multidirectional flow along the direction of the field. (2) Those in which the surface charge pattern varies parallel to the field; this pattern generates recirculating cellular flow, and thus causes motion both parallel and perpendicular to the external field. Measurements of both of these flows agree well with theory in the limit of thin double layers and low surface potential.

PACS numbers: 47.54.+r, 47.65.+a, 61.20.Lc, 82.45.+z

Microfluidic devices are important in a range of applications: microanalytical systems for genomics and proteomics, research tools for cell biology, and low-inventory chemical synthesis. Developments in microfluidics will require sophisticated methods for handling fluids in the low Reynolds number $(\mathrm{Re})$ regime that is imposed by the dimensions of the microchannels in these devices. Electrically driven flow [electro-osmotic flow (EOF)] is an alternative to pressure driven flow in microchannels, but has been used only for the simplest case, that is, for flow driven by uniformly charged channel walls. In this Letter, we describe EOF driven by surface charge patterned on the channel walls [1]. The geometries that we explore demonstrate two new types of fluid behavior at low Reynolds number: multidirectional flow along the axis of the channel and the applied field, and controlled cellular flow with bulk motion both perpendicular and parallel to the axis of the channel and the applied field. We believe that these new types of flows offer opportunities for fine control of fluid motion and chemical transport in microfluidic devices, and also suggest the use of fluids to drive micromechanical elements via shear-generated torques.

The experimental challenge of patterning surfaces inside microstructures has hindered the investigation of EOF in the presence of patterned surface charge. For this study, we used two soft lithographic techniques [2] to solve this problem in patterning. We used laminar flow patterning [3] to generate longitudinal patterns, and a modified version of micromolding in capillaries to generate transverse patterns [4]. In both cases, the electrostatic adsorption of organic polymers having charged side groups [poly(ions)] [5] onto the inside walls of the channels generated the required regions of positive and negative surface charge, and ensured that these regions had charge densities of similar magnitude [6].

Electro-osmotic flow [7] uses an externally applied electric field parallel to a surface with net surface charge density to drive bulk motion in a fluid. The field interacts with the net charge carried by the mobile screening layer (the Debye layer) in the fluid above the surface. A channel with walls having fixed charges thus generates a mobile "sheath" of charge in the fluid. When an electric field is applied, the movement of this sheath transports the bulk of the fluid, as if the walls of the channel were sliding at
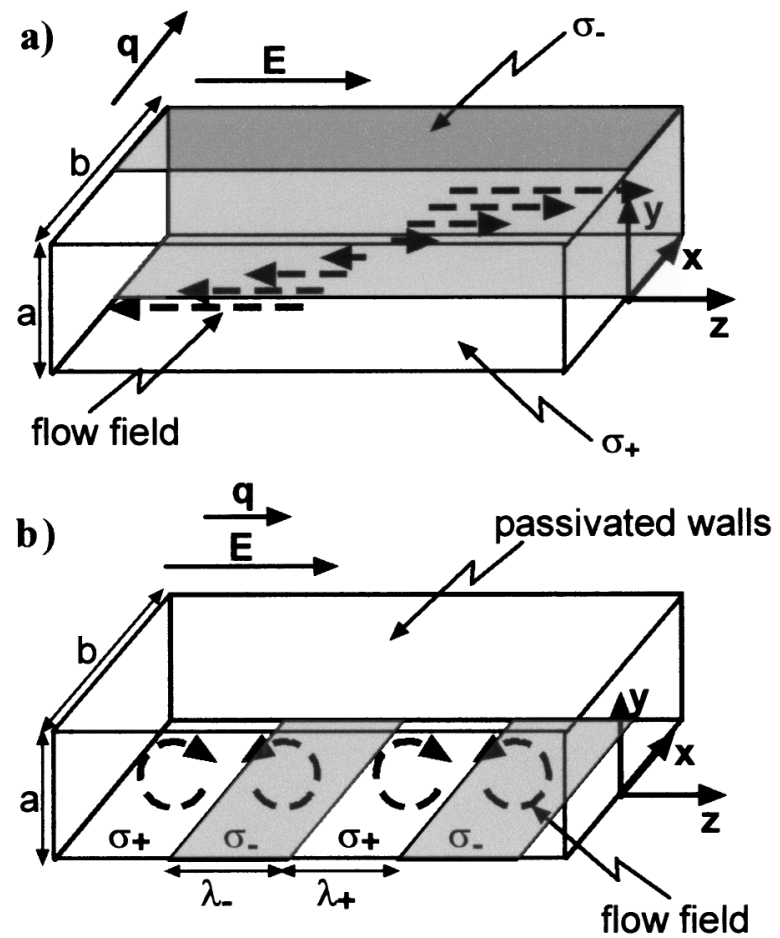

FIG. 1. Schematics of the two patterned geometries studied. $\sigma_{+}$and $\sigma_{-}$represent the surface charge densities. (a) $\mathbf{q} \perp \mathbf{E}$ : Surface charge varying in the direction perpendicular to the applied electric field: positive coating $\left(\sigma_{+}\right)$on one half $(x>0)$ and negative coating $\left(\sigma_{-}\right)$on the other $(x<0)$. q represents the direction of a typical wave vector describing the variations of the charge pattern. The dotted arrows represent the EOF generated in this geometry. (b) $\mathbf{q} \| \mathbf{E}$ : Patterned charge varying parallel to the direction of the applied field. In this study, only one of the four capillary walls is patterned; the other three were treated with a poly(ethylene glycol) (PEG) to reduce their surface charge and the sticking of beads. Dotted arrows represent the EOF in this geometry. 
a velocity of $\mu_{\mathrm{eo}} E$. Here, $\mu_{\mathrm{eo}}$ (speed/field strength) is the electro-osmotic mobility of the charged surface; it is a function of the surface charge density and of the ionic strength of the fluid. $E$ is the magnitude of the applied field parallel to the walls.

In the limit of thin double layers (Debye screening length $=\kappa^{-1} \ll$ pattern and geometric dimensions), the governing equations of EOF in low Reynolds number environments are given by the Stokes equation:

$$
\begin{aligned}
\eta \nabla^{2} \mathbf{v}-\nabla p & =\mathbf{0}, \\
\nabla \cdot \mathbf{v} & =0,
\end{aligned}
$$

with "slip" boundary conditions at the walls of the channel where the Debye layer is moving under the influence of the applied field: $\boldsymbol{v}_{z}\left(x_{0}, y_{0}, z\right)=\mu\left(x_{0}, y_{0}, z\right) E$ [8]. We therefore ignore the details of the flow in the double layer and replace the standard no-slip boundary conditions with the appropriate moving ones. Here, $\mathbf{v}$ is the velocity field, $\mu$ is the electro-osmotic (EO) mobility of the surface, $\eta$ is the viscosity of the fluid, $E$ is the strength of the component of the applied electric field which is parallel to the wall, and $p$ is the pressure field. For low surface or zeta potentials $\left(e \zeta_{0} / k_{B} T<1\right), \mu=\sigma / \eta \kappa$ [7]. Here, $\zeta_{0}$ is the surface potential and $e$ is the electron charge.

We present two cases of patterned EOF in this regime [9]; Fig. 1 shows their geometries. In the first case, the surface pattern varies in a direction perpendicular to the applied electric field, $\mathbf{q} \perp \mathbf{E}$, where $\mathbf{q}$ is a typical wave vector describing variations in the pattern and $\mathbf{E}$ is the field [Fig. 1(a)]. In this geometry, the EOF runs parallel to the field and alternates direction in phase with the surface pattern. In the second case, $\mathbf{q} \| \mathbf{E}$, and the EOF runs in closed cells with the axis of the cells perpendicular to the field and parallel to the patterned surface [Fig. 1(b)] [1].

Over flat surfaces, these two types of flow represent a basis from which flows over arbitrary surface patterns can be constructed [10]. The patterns are made up of regions of adsorbed layers of poly(ions) with surface charge density of opposite sign and similar magnitude. We have worked with channels with $\sim 200 \mu \mathrm{m}$ height and width and pattern features of $\sim 200 \mu \mathrm{m}$ for experimental convenience, but the observed behavior is predicted to be scale independent for systems that satisfy the limit of thin double layers.

As Fig. 1(a) indicates, in the $\mathbf{q} \perp \mathbf{E}$ case, a horizontal cross section of the flow through the middle of the channel $(y=a / 2)$ should be a flow with almost constant shear, and thus similar to the two-dimensional flow generated between two parallel plates separated by $b$ and moving in opposite directions at $\mu_{+} E$ and $\mu_{-} E$. The actual flow is given by the solution of Eqs. (1) with the boundary conditions $v_{z}=\mu_{+} E$ at the walls for $x>0$, and $v_{z}=$ $\mu_{-} E$ at the walls for $x<0$. This purely axial velocity field is approximated by Eq. (2):

$$
\begin{aligned}
\mathbf{v}(x, y)=v_{z} \mathbf{e}_{z}=[ & \left.-\mu_{b} E+(1 / 2)\left(\mu_{+}+\mu_{-}\right) E\right] \mathbf{e}_{z}+(1 / 2)\left(\mu_{+}-\mu_{-}\right) E \\
& \times \sum_{n=1}^{\infty}(1 / n)\{\operatorname{csch}(2 n \pi a / b)[\sinh (n \pi y / b)-\sinh (n \pi(y-2 a) / b) \sin (n \pi x / b)] \\
& +\operatorname{csch}(n \pi b / 2 a) \sinh (n \pi x / 2 a) \sin (n \pi y / 2 a)\} \mathbf{e}_{z} .
\end{aligned}
$$

Here, $\mu_{ \pm}=\left(\sigma_{ \pm} / \eta \kappa\right)$ and $\mu_{b}$ is the electrophoretic mobility of the beads used to trace the flow.

In the $\mathbf{q} \| \mathbf{E}$ case, Fig. 1(b), a vertical cross section of the flow through the middle of the channel $(x=0)$ satisfies a version of the solution proposed by Ajdari, taken in the appropriate limits [1]. Equation (3) shows the predicted stream function for a square-wave form of surface charge density:

$$
\begin{aligned}
\varphi(y, z)=E f_{c}(x, y)-(2 / \pi)\left(\mu_{+}-\mu_{-}\right) E \sum_{n=1}^{\infty} & \left\{n^{-1} \sin \left(\pi q_{n} \lambda_{+}\right)\left[\left(\mu_{+}+\mu_{-}\right) /\left(\mu_{+}-\mu_{-}\right)+\cos \left(q_{n} z\right)\right]\right. \\
& \left.\times\left[c_{1} \sinh \left(q_{n} y\right)+c_{2} y e^{-q_{n} y}+c_{3} y e^{+q_{n} y}\right]\right\} .
\end{aligned}
$$

Here, $q_{n}=2 \pi n / \lambda$, where $\lambda=\lambda_{+}+\lambda_{-}$is the periodicity of the pattern, and $\lambda_{+}$and $\lambda_{-}$are the widths of the positive and negative bands, respectively. The function $f_{c}(x, y)$ is used to account for the nonzero mobilities of the unpatterned walls and of the beads used to trace the flow $f_{c}(x, y)=y\left[\mu_{\text {beads }}+\mu_{\text {walls }} f_{w}(x, y)\right]$, where

$$
\begin{gathered}
f_{w}(x, y)=\sum_{n=1}^{\infty}\{\operatorname{csch}(n \pi h / w) \sin (n \pi y / b)[\sinh (n \pi x / b)-\sinh (n \pi(x-a) / b)] \\
+\operatorname{csch}(n \pi b / a) \sinh (n \pi y / a) \sin (n \pi x / a)\} .
\end{gathered}
$$

The values of the coefficients $c_{1}, c_{2}$, and $c_{3}$ are

$$
\begin{aligned}
& c_{1}=\left(q_{n} a\right)^{2} / q_{n}\left[1+2\left(q_{n} a\right)^{2}-\cosh \left(2 q_{n} a\right)\right], \\
& c_{2}=\left[e^{2 q_{n} a}-\left(q_{n} a\right)^{2}-1\right] / 2\left[1+2\left(q_{n} a\right)^{2}-\cosh \left(2 q_{n} a\right)\right], \\
& c_{3}=\frac{\left[\sinh \left(q_{n} a\right)-\cosh \left(q_{n} a\right)\right]\left[q_{n} h \cosh \left(q_{n} a\right)+\left(q_{n} a-1\right)\left(q_{n} a\right)\right]}{1+2\left(q_{n} a\right)^{2}-\cosh \left(2 q_{n} a\right)} .
\end{aligned}
$$


The velocity field can be obtained from $\varphi$ in the standard way: $v_{y}=(\partial \varphi / \partial z)$ and $v_{z}=-(\partial \varphi / \partial y)$.

Figure 2(a) shows an example of bidirectional flow generated by the $\mathbf{q} \perp \mathbf{E}$ pattern shown in Fig. 1(a). We imaged the flow using $2 \mu \mathrm{m}$, fluorescent microspheres with poly(ethylene glycol) (PEG) modified surfaces. Figure 2(b) shows a plot of the velocities taken from data of the type shown in Fig. 2(a) and fit with Eq. (2) evaluated at $y=130 \mu \mathrm{m}$ and using 50 terms in the sum. We have no independent measure of the $\mu_{+}$and $\mu_{-}$ values generated by our patterning technique, so these values were used as adjustable parameters in Eq. (2). The residual electrophoretic mobility of the tracer beads was measured independently: $\mu_{b}=-0.45(\mu \mathrm{m} / \mathrm{s}) /(\mathrm{V} / \mathrm{cm})$. Regression led to $\mu_{+}=2.9(\mu \mathrm{m} / \mathrm{s}) /(\mathrm{V} / \mathrm{cm})$ and $\mu_{-}=-3.7(\mu \mathrm{m} / \mathrm{s}) /(\mathrm{V} / \mathrm{cm})$. These values of $\mu_{+}$and
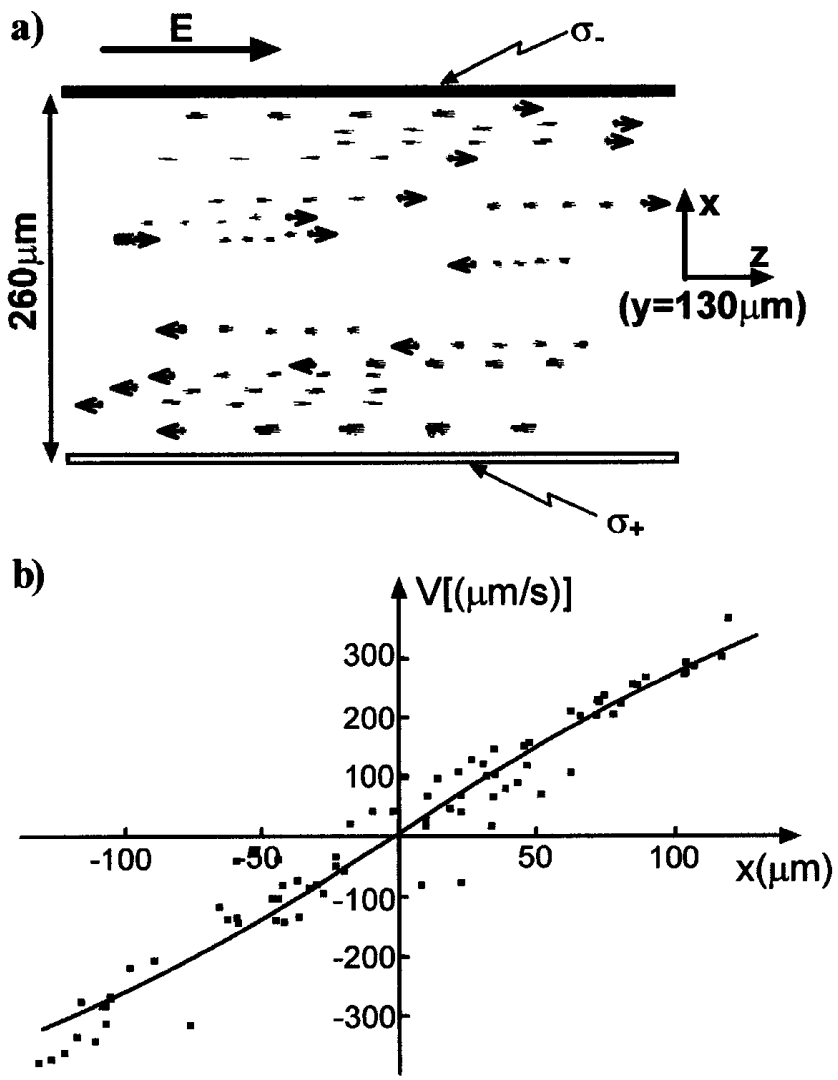

FIG. 2. $\quad \mathbf{q} \perp \mathbf{E}$ : Experiment and prediction. (a) Bidirectional EOF in a channel patterned as is shown in Fig. 1(a). The $260 \mu \mathrm{m} \times 260 \mu \mathrm{m}$ channel was viewed from the top (along the negative $y$ axis) through a $25 \times$ lens with fluorescence filters. The focal plane was located at $y=130 \mu \mathrm{m}$. PEGfunctionalized $2 \mu \mathrm{m}$ fluorescent beads were used as flow tracers. Arrows were added to indicate direction. The applied field (along z) was $95 \mathrm{~V} / \mathrm{cm}$. (b) Fit of the bidirectional flow profile to Eq. (2): The solid dots represent axial $(z)$ velocities taken from images like those pictured in (a). The velocities are plotted against the $x$ position of the bead. The scatter in the data is dominated by variations in the mobility of the tracer beads and small Poiseuille flows which were present in the channel. The solid line is an optimized fit generated with Eq. (2). $\mu_{-}$are approximately $60 \%$ of those found in channels coated uniformly with the same poly(ion)s $(4.8$ and -5.8$)$. This discrepancy may reflect desorption of polymers from one region and adsorption on the other during or after patterning.
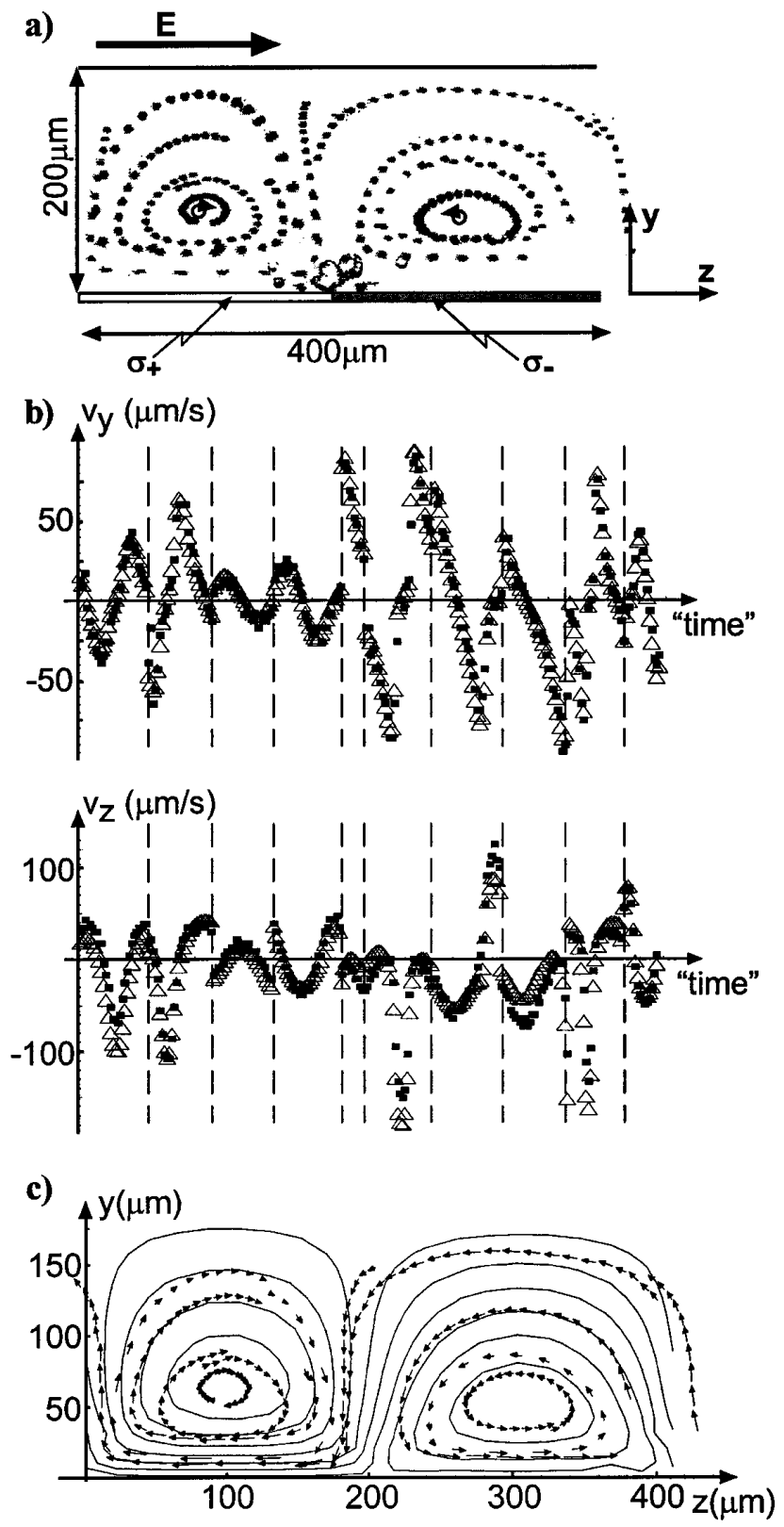

FIG. 3. $\quad \mathbf{q} \| \mathbf{E}$ : Experiment and prediction. (a) A series of trajectories over a single period of a pattern of the type illustrated in Fig. 1(b). The channel $(200 \mu \mathrm{m} \times 200 \mu \mathrm{m})$ was viewed from the side (along the $x$ axis) with a $25 \times$ lens. The focal plane was located near the center of the channel $(x \sim 0 \mu \mathrm{m})$. (b) Comparison of each component of the measured velocity field $(\square)$ with predictions $(\triangle)$ made by evaluating Eq. (3) at the measured $(y, z)$ positions and plotted against an arbitrary time scale. Dashed lines separate segments associated with independent trajectories. (c) Comparison of the experimental velocities measured along the trajectories pictured in (a) (arrows) with the stream function calculated with Eq. (3) (solid lines). 
Figure 3(a) shows a series of bead trajectories over lines of positive and negative surface charge on the floor of the channel as illustrated in Fig. 1(b). The globular objects at the patterned surface near the junction of positively and negatively charged regions are beads stuck to the glass slide out of the focal plane. The beads stuck predominantly in this region, where the flow field brings them toward the surface, into a stagnation point. With the direction of the field reversed (along the positive $z$ axis), the sticking occurred at the minus-plus junctions.

Figure 3(b) shows comparisons of the components of the velocity field extracted from the trajectories in 3(a) with values calculated using the stream function in Eq. (3) (using 30 terms in the sums). Dashed lines separate data from independent trajectories. We used $v_{y}(y, z)$, which is independent of the mobility of the tracer beads $\left(\mu_{b}\right)$ and of the unpatterned walls $\left(\mu_{w}\right)$, to adjust the values $\mu_{+}$and $\mu_{-}$in Eq. (3): $\mu_{+}=1.9(\mu \mathrm{m} / \mathrm{s}) /(\mathrm{V} / \mathrm{cm})$ and $\mu_{-}=-1.9(\mu \mathrm{m} / \mathrm{s}) /(\mathrm{V} / \mathrm{cm})$. A value of $\mu_{w}=$ $-0.55(\mu \mathrm{m} / \mathrm{s}) /(\mathrm{V} / \mathrm{cm})$ gave the best fit of $v_{z}(y, z)$. We determined all other parameters in Eq. (3) experimentally: $\lambda_{+}=200 \mu \mathrm{m}$ and $\lambda_{-}=212 \mu \mathrm{m}, \quad E=95 \mathrm{~V} / \mathrm{cm}$, $\mu_{\text {bead }}=-0.45(\mu \mathrm{m} / \mathrm{s}) /(\mathrm{V} / \mathrm{cm})$. The variable fidelity of the fit for different segments of the plot of $v_{z}$ is a reflection of differences in the mobilities of individual beads.

This work demonstrates two examples of the rich fluid behavior that a surface with a patterned surface charge can induce in EOF. These results are restricted to the thin double layer limit. The two types of patterns demonstrated here act as a basis from which more general threedimensional flows may be generated in microfluidic environments (e.g., helical flows over bands of positive and negative charged bands running diagonally to the axis of the channel [11]). A distinguishing characteristic of recirculating EOF is that movement is generated perpendicular to the applied field. This motion could be particularly useful in systems in which torque must be generated on a micron scale, and might also serve as a mixer for EO driven flows. Stationary points in these recirculating flows may also be useful for the manipulation of macromolecules and cells in a fluid environment.

We also note that EOF generated by surfaces with inhomogeneous charge distributions, and by the motion of inhomogeneously charged particles undergoing electrophoresis, can be described within the same theoretical framework; the system presented here describing EOF in the presence of stationary, charged surfaces therefore provides information about charged, mobile particles in fluids [1].

We are grateful to Professor A. Ajdari (Ecole Supérieure de Physique et Chimie Industrielles de Paris) and Professor H. Stone (DEAS, Harvard University) for helpful discussions. This work was supported by DARPA: NSF ECS-9729405, NIH: GM51559, and NSF: DMR-9809363 MRSEC. A. D. S. was supported by NIH Molecular Biophysics Training Grant No. 5T32GM08313-10. M. W. was supported by the German Academic Exchange Service (DAAD). P. J. A. K. was supported by Netherlands Organization for Scientific Research (NWO).

*To whom correspondence should be addressed.

[1] For a theoretical treatment of EOF in the presence of patterned surface charge see A. Ajdari, Phys. Rev. Lett. 75, 755 (1995). For a treatment of the electro-osmosis of inhomogeneously charged particles see J. Anderson, J. Colloid Interface Sci. 105, 45 (1985); P. Long and A. Ajdari, Phys. Rev. Lett. 81, 1529 (1998).

[2] Y. Xia and G. M. Whitesides, Angew. Chem., Int. Ed. Engl. 37, 550 (1998).

[3] P. K. Kenis, R. F. Ismagilov, and G. M. Whitesides, Science 285, 83 (1999).

[4] N. L. Jeon, I. S. Chio, B. Xu, and G. M. Whitesides, Adv. Mater. 11, 946 (1999).

[5] G. Decher, Compr. Supramol. Chem. 9, 507 (1996); P. T. Hammond and G. M. Whitesides, Macromolecules 28, 7569 (1995).

[6] Experimental details to be published later.

[7] W. B. Russel, D. A. Saville, and W. R. Schowalter, Colloidal Dispersions (Cambridge University Press, Cambridge, 1989); Capillary Electrophoresis: Theory and Practice, edited by P.D. Grossman and J. C. Colburn (Academic Press, San Diego, 1992).

[8] D. A. Saville, Annu. Rev. Fluid Mech. 9, 321 (1977).

[9] For the buffer system used, the molarity of ionic species was $\sim 20 \mathrm{mM}$, making the thickness of the double layer $\sim 1-2 \mathrm{~nm}$. Thus, $\kappa^{-1} \ll$ dimensions of channels and charge patterns. $e \zeta_{0} / k_{B} T \sim 1$ for our surfaces.

[10] Superpositions of patterned surface charge and periodic topographical patterns are considered in A. Ajdari, Phys. Rev. E 53, 4996 (1996).

[11] Unpublished results from this laboratory. 